\section{Chronisch spontane Urtrikaria: parasitäre Ursachen eher selten}

\author{
Bei den meisten Patienten mit einer chronisch spontanen Urtikaria bleibt die \\ Ursache im Dunkeln. Eine erste systematische Übersichtsarbeit beschäftigt \\ sich mit parasitären Auslösern und analysiert Prävalenz sowie mögliche \\ Pathomechanismen.
}

$D^{a}$ er Zusammenhang zwischen Parasiteninfektionen (PI) und einer chronisch spontanen Urtikaria (CSU) wurde in mehreren Studien untersucht. Ein internationales Allergologenteam hat nun 39 Studien zur Prävalenz einer PI bei CSU-Patienten systematisch untersucht. In zwei Dritteln dieser Studien lagen die Infektionsraten bei $10 \%$ oder weniger, insgesamt wurden Raten von $0-75,4 \%$ bei Erwachsenen und $0-37,8 \%$ bei Kindern gefunden. Im Vergleich zu Kontrollen hatten CSU-Patienten häufiger eine Protozoeninfektion, wurden häufiger seropositiv unter anderem auf Toxocara canis getestet und hatten häufiger eine Anisakis-simplex-Sensibilisierung.
In weiteren 21 Studien, die den Zusammenhang zwischen dem Verschwinden der CSU-Symptome und einer Behandlung mit antiparasitär wirkenden Mitteln untersuchten, lagen die Erfolgsquoten zwischen $0 \%$ und $100 \%$. In der Zusammenschau der Studien profitierten $35,7 \%$ von insgesamt 269 Patienten von der Antiparasiten-Therapie.

Der pathogenetische Zusammenhang zwischen PI und CSU bleibt allerdings hypothetisch. Vermutlich spielt parasitenspezifisches IgE, vor allem von Helminthen und Protozoen, eine Rolle. Die Immunglobuline binden zusammen mit dem Parasitenantigen die FceRI von Mastzellen, die daraufhin degranulie- ren und die CSU-Symptome zum Beispiel durch den Mediator Histamin auslösen. Darüber hinaus existieren weitere Hypothesen zu einer Beteiligung von PI-getriggerten Th2-Zytokinen, Eosinophilen oder zirkulierenden Immunkomplexen, die die Mastzellen ebenfalls aktivieren.

Fazit: Parasitäre Infektionen können aufgrund vermutlich verschiedener immunologischer Mechanismen eine CSU auslösen. Parasitäre Infektionen sind bei uns allerdings eher selten. Ein Zusammenhang gilt erst dann als gesichert, wenn nach Parasiten-Eradikation auch die CSU verschwunden ist. Hinweise auf eine mögliche PI liefern gastrointestinale Symptome, anamnestisch bekannte Parasiteninfektionen, Reisen in Länder mit niedrigem Hygienestandard sowie eine anderweitig nicht erklärliche Eosinophilie.

Dr. Barbara Kreutzkamp

Kolkhir et al. Chronic spontaneous urticaria and internal parasites - a systematic review. Allergy 2016; 71: 308-322

\title{
Ein Sechsjähriger mit Wimpernläusen
}

Ein sechsjähriger Junge wurde in der Universitätsklinik von Catania wegen anhaltender Rötung und Juckreiz im Bereich der Augenlider (Abb. A) vorgestellt. Unter der Diagnose einer atopischen Dermatitis war er bereits zwei Wochen lang mit kortikoidhaltigen Salben und Antihistaminika erfolglos behandelt worden. Bei der Dermatoskopie erkannte man mehrere Filzläuse (Phthirus pubis) sowie ovaläre Nissen an den Wimpern (Abb. B). Unter der Diagnose einer Phthiriasis palpebrarum wurde der Junge mit 5\%iger gelber Quecksilberoxidsalbe viermal am Tag über zwei Wochen hinweg behandelt, worunter sich die Symptomatik vollständig zurückbildete.
Die Wimpern sind bei Kindern ein übliches Habitat für die Filzlaus, nachdem sich in diesem Alter an anderen Körperstellen noch kein Terminalhaar findet. Die Läuse werden üblicherweise durch engen Kontakt mit befallenen Erwachsenen übertragen. Bedenkt man die Lokalisation bei Erwachsenen, so keimen heutzutage natürlich dunkle Gedanken über den Übertragungsmechanismus auf. Die Diagnose ist manchmal optisch nicht leicht zu stellen, da sich die Läuse tief in den Lidrand eingraben und man wegen der Rötung im Bereich der Augen zunächst an eine atopische Dermatitis oder eine allergische Konjunktivitis denkt. Prof. Hermann S. FüeßI

Micali G et al. Phthiriasis palpebrarum in a child. N Engl J Med 2015; 373: e35
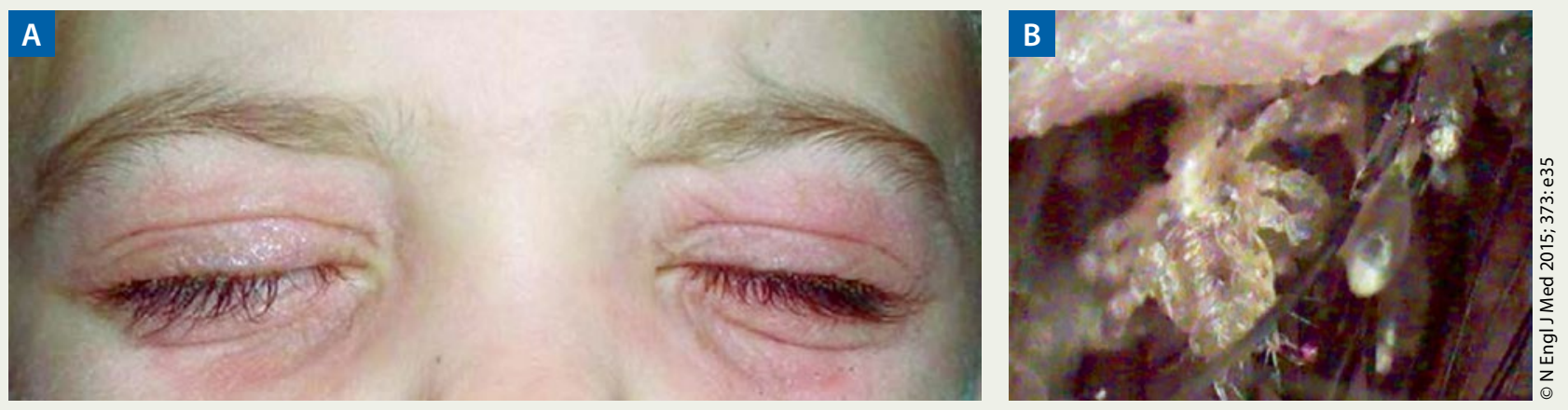

A: Beidseitige Rötung im Bereich der Augenlider, B: Filzläuse (Phthirus pubis) und ovaläre Nissen an den Wimpern. 